\title{
Transient impurity events in JET with the new ITER-like wall
}

\author{
M.Sertoli $^{1 *}$, J.C. Flannegan ${ }^{2}$, A. Cackett $^{2}$, E. Hodille ${ }^{3}$, P. \\ ${\text { de } \text { Vries }^{4} \text {, I.H. Coffey }}^{5}$, B. Sieglin ${ }^{1}$, S. Marsen ${ }^{6}$, S. \\ Brezinsek $^{7}$, G.F. Matthews ${ }^{2}$, J.W. Coenen ${ }^{7}$ and \\ JET-EFDA Contributors \# \\ JET-EFDA Culham Science Centre, Abington, OX14 3DB, UK \\ ${ }^{1}$ Max-Planck-Institut fr Plasmaphysik, EURATOM Association, 85748 \\ Garching, Germany \\ ${ }^{2}$ Euratom/CCFE Fusion Association, Culham Science Centre, Abingdon, OX14 \\ 3DB, UK \\ ${ }^{3}$ Universite Claude Bernard Lyon I, 69622 Villeurbanne, France \\ ${ }^{4}$ FOM institute DIFFER, Association EURATOM-FOM, P.O. Box 1207, \\ 3440BE, Nieuwegein, Netherlands \\ ${ }^{5}$ Queen's University, Belfast, BT7 1NN, UK \\ ${ }^{6}$ Max-Planck-Institut fr Plasmaphysik, EURATOM Association, 7491 \\ Greifswald, Germany \\ ${ }^{7}$ Forschungszentrum Juelich GmbH, EURATOM Association, 52425 Juelich, \\ Germany \\ \# See the Appendix of F. Romanelli et al., Proceedings of the 24th IAEA \\ Fusion Energy Conference 2012, San Diego, US \\ E-mail: marco.sertoli@ipp.mpg.de
}

\begin{abstract}
Transient impurity events leading to unexpected increase in radiated power have been studied in JET from the installation of the ITER-like wall. A total of 1800 events over 2800 plasma discharges have been detected. None have led to permanent changes in plasma conditions. Of all events $60 \%$ show traces of $\mathrm{W}, 25 \%$ of either $\mathrm{Ni}, \mathrm{Fe}$ or $\mathrm{Cr}$ from either Inconel or steel structures. They occur mainly in diverted magnetic configuration, independently of strike-point position. The effect of disruptions on dust redistribution has been investigated using the Thomson scattering diagnostic and correlated with TIE occurrence. The number of dust events detected increases with disruption force and, in comparison to the full-C wall, the amount of dust mobilized is found to be about an order of magnitude lower. Their time evolution correlates well with that of the transient impurity events.
\end{abstract}

PACS numbers:

Keywords: tokamaks, dust, impurity, disruption, VUV spectroscopy

\section{Introduction}

The understanding of dust production and control in future tokamaks such as ITER is a major concern not only for safety reasons and for diagnostic interpretation (see e.g. [1, 2, 3]), but also for reliable plasma operation. Depending on their composition and size, if such particles reach the plasma core they can perturb it with intense radiation spikes which have been seen to lead to MARFE (stranding for Multifaceted 
Asymmetric Radiation From the Edge) instabilities or disruptions, especially in long pulse operation [4].

This contribution reports on the study of the occurrence of such transient impurity events (TIEs, also called UFOs) in JET from the installation of the new ITER-Like wall [5. Such events are visible as sharp increases in the total radiated power similar to what caused by laser ablation experiments. All plasma phases with $I_{p}>1(M A)$ from pulse number JPN 81000 have been considered. The impurities responsible have been studied using vacuum-ultra-violet (VUV) spectroscopy. Correlations with auxiliary heating power, plasma geometry (limiter or diverted magnetic configurations, as well as positions of the strike-points) external causes such as arcs from the lower-hybrid antennas or reciprocating probe plunges and disruptions have been investigated. This contribution reports on the time evolution of these events during the first campaigns with the ILW, on the correlation with plasma geometry and on the elements responsible (section 2). Moreover, studies of the dust mobilized by disruptions examined using the high resolution Thomson scattering (HRTS) diagnostic and their correlations with TIEs are reported (section 3).

\section{Transient impurity event analysis}

The occurrence of transient impurity events has been analysed in all phases of plasma discharges with current $I_{p}>1 M A$. A total of 2792 discharges have been analysed for a total of 1781 events detected starting from JPN 81000. The events have been selected by searching for a sharp increase in the total radiated power similar to what commonly observed in impurity injection experiments. None of these events have been seen to lead to permanent changes in plasma conditions (e.g. permanent loss in confinement). TIE-induced disruptions are not reported in this contribution since the analysis of cause-and-effect requires more in-depth investigations than what can be reported in here.

For each event, the values and variations of the ohmic and auxiliary heating power, plasma current, plasma geometry (high- or low-field-side limiters, strike point positions for diverted plasmas), excursion in radiated power and total injected deuterium rate have been tabulated. In order to correctly normalize the event distributions, a database containing the total plasma operation time (in steps of $10 \mathrm{~ms}$ ) for each geometrical configuration and each correlation quantity listed above has been created.

A new database containing the total plasma operation time (in steps of $10 \mathrm{~ms}$ ) for each geometrical configuration and each correlation quantity listed above provides the normalization parameters for the event distributions. The probability of TIE occurrence is therefore given in \# of events / operation time, i.e. as a frequency in units of $\mathrm{Hz}$. Its error bars are evaluated through propagation of the statistical error of the number of events, equal to its square root.

\subsection{Evolution during the campaign}

The evolution in time of the probability of TIE-occurrence is shown in figure 1 . The discharges have been binned according to pulse number (bin size $=200$ ), but each bin is independently normalized to the total plasma time in that bin. The distribution shows two main jumps in probability at $J P N \sim 81501$ and $J P N \sim 83301$, sitting on an almost constant background value $\sim 0.03 \mathrm{~Hz}$, i.e. 3 events every 100 seconds of plasma operation. 


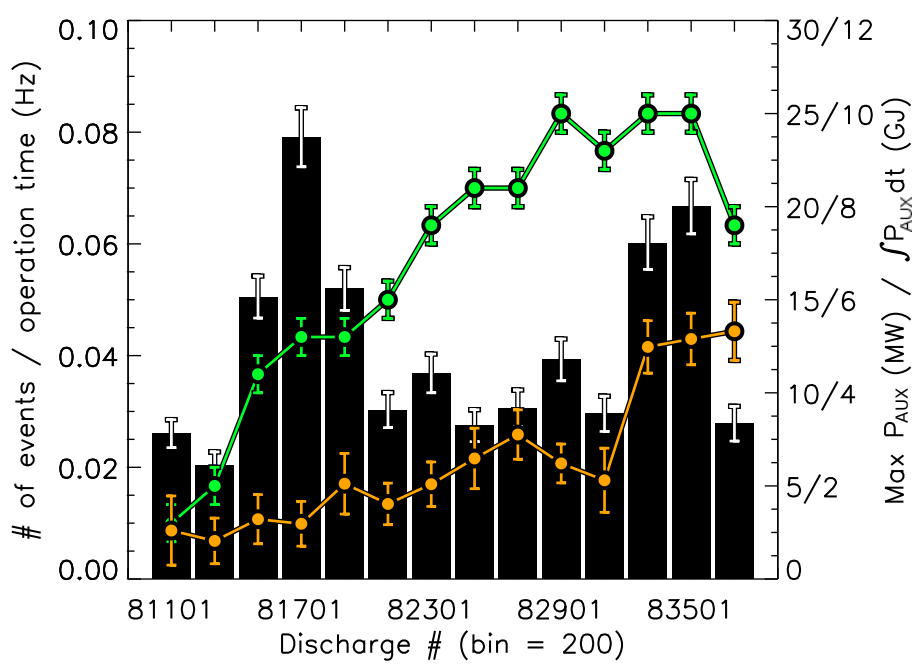

Figure 1. Time evolution of the TIE probability (black with white error bars) and of the maximum heating power ( $M a x P_{A U X}$ in green) and total injected energy ( $\int P_{A U X} d t$ in orange).

The first increase $(J P N=81501 \pm 100)$ in the campaign sets in when additional power by neutral-beam injection (NBI) was available and routinely used in the discharges. In the phases before, almost pure ohmic or ion cyclotron resonance frequency (ICRF) heated discharges had been performed. The maximum heating power (in green in figure 1) undergoes a sharp increase from $<5$ to $\sim 13 \mathrm{MW}$, more than $80 \%$ of which is due to NBI. This suggests a correlation with input power with threshold at $>4 M W$ which is the typical power threshold for type I ELMy (edge localized modes) H-mode. Since most TIEs occur in diverted configuration (see section 2.2 , this is most probably a correlation with power delivered to the divertor. This statement requires in-depth analysis and cross-correlation with other quantities as well as space for sufficient discussion, so it will be left as a speculation in this contribution and discussed elsewhere.

The second increase in event probability $(J P N=83301 \pm 100)$ occurs instead when the total injected energy is increased almost of a factor 3 . Since the normalization of the number of events has been performed over total plasma-time, this increase is probably caused by simple cumulative effects due to higher fraction of total time operated at high input power. More in this regard is discussed in section 3.

\subsection{Geometrical dependencies and impurity identification}

The probability of TIEs occurring in limited plasmas is very low $(\leq 10 \mathrm{mHz})$ and only $\sim 7 \%$ of all events occur in this configuration. Most are generated in divertor configuration, the probability increasing to $\sim 40-50 \mathrm{mHz}$, almost independently of strike point configuration (figure 2). Configurations with outer strike point on stack $A$ of tile 5 account for only 10 events so the probability is in this case subject to large errors (black circles on coloured blobs whose size is proportional to the TIEprobability). Similarly, configurations with outer strike point on tile 7 account instead for only 6 events, while the outer strike point has never been positioned on tile 8 , so 


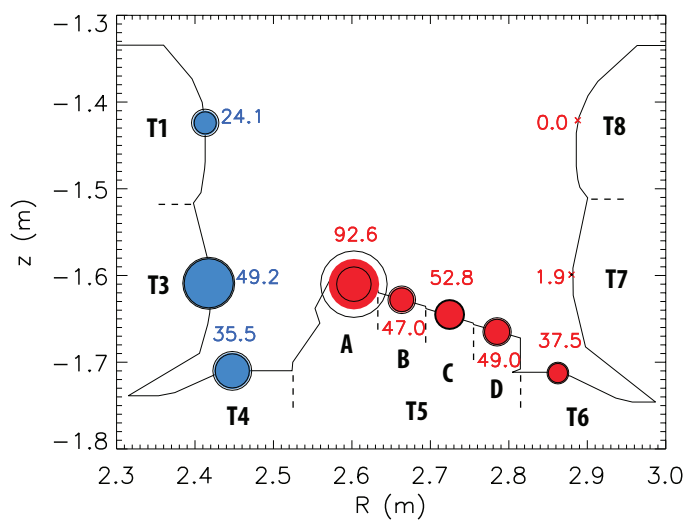

Figure 2. TIE probability (in $m H z$ ) depending on strike point position for the inner (blue) and for the outer (red) strike points. The size of the coloured blobs are proportional to the TIE-probability written close to them with the same colour. Tile names are labeled in black $(T \#)$, stacks of tile 5 with letters.

no statistics is available.

Configuration with $[$ inner, outer $]$ strike points on tiles $[3,5]$ has been the most commonly used with the ILW, followed by $[4,6],[1,5],[3,7],[3,6]$ and $[4,5]$ used for respectively $25 \%, 17 \%, 15 \%, 7 \%, 4 \%$ of the operation time spent in configuration $[3,5]$. Since most high power scenarios were performed in configuration $[3,5]$, its $\sim 30 \%$ higher probability with respect to $[4,6]$ is most probably due the power dependence of TIEs. Due to the fact that the normalization has been performed over total plasma-time in each specific configuration, the TIE-probability shown in these plots will increase with the fraction of total time spent at higher heating powers.

Configurations with the outer strike point on vertical tile 7 seem to be less prone to TIE-occurrence than those with the outer strike point on the horizontal tiles 5 or $6(1.9 \mathrm{mHz}$ vs. $40-50 \mathrm{mHz})$. Since the horizontal part of the divertor is a deposition zone for material coming from the main chamber, dust which accumulates in this region could partly be the cause of the occurrence of TIEs. On the other hand, these results are biased by the low externally injected heating power and energy in this configuration, never exceeding $\sim 5 M W$ and $\sim 0.05 G J$ respectively, versus $\sim 25 \mathrm{MW}$ and $\sim 12 \mathrm{GJ}$ delivered in configuration [3,5]. More study is therefore required to better understand these issues.

For each event, the elements responsible have been analysed using two VUV spectrometers, measuring respectively in range $[10,100] \mathrm{nm}$ and close to $5 \mathrm{~nm}$. The first one measures spectral lines of $\mathrm{Ni}, \mathrm{Fe}, \mathrm{Cr}, \mathrm{Al}$ and $\mathrm{Cu}$, and can provide an indication of tungsten. The presence of this element has been further cross-checked with the second spectrometer which measures the quasi-continuum spectral features of tungsten ions $W^{27+}-W^{35+}[6]$. This spectrometer is not subject to spectral contamination from other elements within the probed spectral range, so provides a trustworthy measurement of the presence $\mathrm{W}$. An attempt has also been done to detect Be or C through visible spectroscopy [7].

Since both stainless steel (material of the support structures) and Inconel (support structure of the divertor $\mathrm{W}$ mono-block tiles) are made up of $\mathrm{Ni}, \mathrm{Fe}$ and $\mathrm{Cr}$ (although 
in different percentages), dust particles from these parent structures could lead to events of either of these elements. The elements seen have thus been sub-divided into three groups: $\mathrm{W}-\mathrm{Ni} / \mathrm{Fe} / \mathrm{Cr}$ - Others. The latter group accounts for all those events for which neither of the first four elements have been detected. These events could be due to light impurities not distinguishable through VUV or visible spectroscopy (e.g. Be or $\mathrm{C}$ ) or to the elements listed above but in conditions unfavorable for their detection by spectroscopic means (e.g. too low photon flux for detection through the VUV spectrometers). Data from the visible divertor spectroscopy, sensitive to C and $\mathrm{Be}$, show only few of the events present in the database. Identification of the composition and origin of the source have so far been unsuccessful [7].

All divertor configurations show approximately the same element dependency: $\sim 60 \%$ of all events show traces of $W, \sim 20 \%$ of either $\mathrm{Ni}, \mathrm{Fe}$ or $\mathrm{Cr}$ and $\sim 20 \%$ are of unknown origin. About $90 \%$ of the $W$ events do not show any traces of $\mathrm{Ni}, \mathrm{Fe}$ or Cr. Limiter TIEs show instead a lower probability of $\mathrm{W}(\sim 50 \%)$, and increase of $\mathrm{Ni} / \mathrm{Fe} / \mathrm{Cr}(\sim 35 \%)$ and of unknown elements $(\sim 25 \%)$. In limited plasmas, W could originate from $\mathrm{W}$-coated neutral beam shine-through protection tiles as well as from few outer poloidal limiters, recessed inner limiter tiles and restraint ring protection tiles. At the moment, there is no clear indication of which of these sources could be the major contributor to $\mathrm{W}$ limiter-TIEs.

Reciprocating probe (RCP) plunges have shown to be the cause of 15 events $(<1 \%)$, while arcs at the lower hybrid $(\mathrm{LH})$ antennas are responsible for 7 events $(<0.5 \%)$. All LH-arc events and $80 \%$ of the RCP ones show traces of $\mathrm{Fe}$, consistent with the material of the LH launcher (stainless steel) and an indication that material from the probe shaft (Inconel and steel) could have been scratched away or eroded during the plunges.

\section{Disruptions and HRTS dust analysis}

Since dust could be one of the possible causes of TIEs, it is important to try and correlate the results obtained in the present analysis with studies performed with other

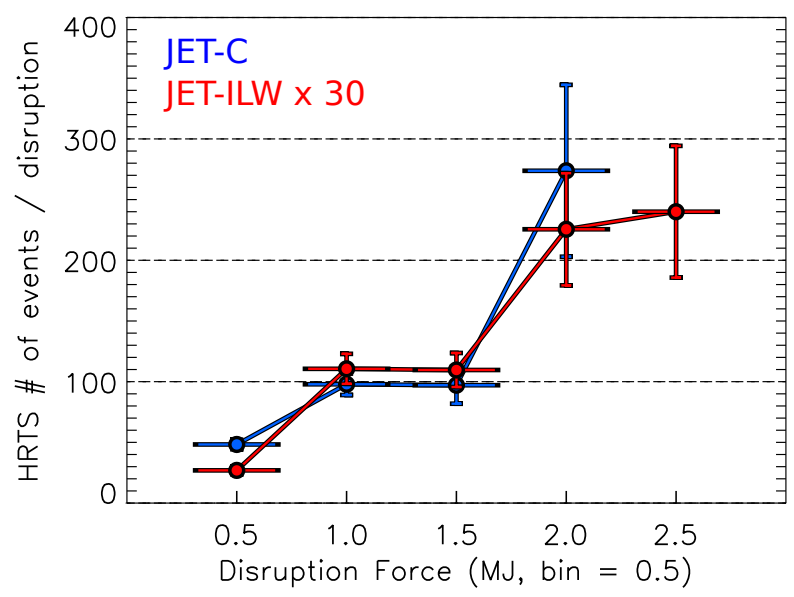

Figure 3. HRTS number of dust events vs. disruption force, normalized to the number of disruptions in each bin for the JET-C wall (blue) and for the JET-ILW (red), the latter multiplied by a factor 30 . 


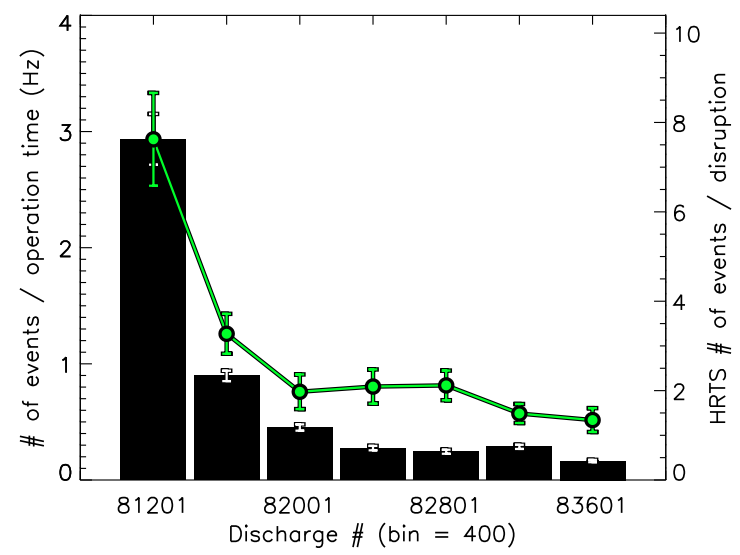

Figure 4. Time evolution of the TIE probability (black with white error bars) re-binned with respect to figure 1 and normalized to total plasma time with $P_{E X T}>4 M W$. In green the HRTS \# of dust events per disruption.

complementary diagnostics. This has been done by analysing the effects of disruptions on the redistribution of dust particles using the High Resolution Thomson Scattering (HRTS). This diagnostic measures the temperature and density of the plasma electrons by analysing the light scattered from a $3 J, 20 n s$ Nd:YAG laser 8 . Following a disruption, light scattered from dust particles (through Mie scattering) that have been heated or ablated by the laser beam is also seen 9. The HRTS dust signal is analysed using the method outlined in 9, which involves fitting a pair of delayed Gaussians to each raw signal. Here, an additional filtering step is applied in order to discard fits to noise (based on the signal amplitude and the goodness of fit statistics). In this way, both the number of dust events and their magnitude can be extracted.

Correlation with disruption force reveals that the number of detected dust particles increases strongly with disruption force (figure 3 ) and confirms the results shown in [10. Moreover, it indicates that disruptions may stir-up dust particles present in the machine. The shape of this distribution in the ILW (red in figure 3p is very similar to what encountered in the JET-C (blue), but more than an order of magnitude lower (multipled by a factor 30 in figure).

The number of events detected by the HRTS diagnostic has been seen to decrease constantly during the experimental campaigns with the ILW, dropping of a factor $\sim 4$ from the start of the first plasmas to the end of the campaign. This is consistent with visual studies on the divertor tiles which show debris on the divertor tiles at octant 8 before the first plasma (23 August 2011) which are not found at later inspections more than 50000 operational seconds later (11 May 2012). The debris from machining of the ILW is probably the largest source of such dust particles. This clean-up phase has also been observed in-situ by optical spectroscopy in the visible range with reduction of carbon, oxygen and other impurities in the first phase of operation [11.

The time-evolution of TIEs as shown in figure 1 does not reproduce this evolution, but this is due to wrong normalization. Since a $\sim 4 M W$ threshold has been observed 
in TIE generation, normalizing the number of TIE events to the total plasma time with external heating power greater than this threshold one obtains a very similar trend as observed by HRTS (figure 4). The dust observed by HRTS after a disruption and the TIEs seen during plasma operation could therefore be the same dust particles, but visualized in two different manners. This suggest that the dust initially present in the machine before the first plasma (or generated before $J P N=81000$ for the TIE database) has been slowly eroded, or ablated when interacting with the hot plasma [12], or transported to remote, inaccessible areas of the machine. The occurrence of TIEs could be then understood in the following way: when entering the plasma core, the dust particles will ablate and lose part of its mass which will be then re-deposited in other regions of the machine. This dilution of dust would then lead to a lower probability of detectable TIEs as well as lower number of detectable dust particles from the HRTS diagnostic).

More study will be necessary to confirm/confute this hypothesis. Simulations of dust penetration probability (in the core plasma) from different regions of the torus as well as more study on the origin of the dust particles detected by the HRTS diagnostic may clarify these issues.

\section{Conclusions and Outlook}

The transient impurity events observed in JET from the installation of the ILW occur mainly in the divertor configuration, independently of strike-point position. None of these events has lead to permanent changes in plasma conditions such as long lasting confinement degradation. Their occurrence shows a input power threshold $>4 M W$ which is close to the required auxiliary power required for H-mode. Further analysis will be necessary to understand what the divertor conditions are and what role they play in TIE generation. Most events show traces of $W$ but $N i, \mathrm{Fe}$ or $\mathrm{Cr}$ are often seen. $\sim 20 \%$ of all events could not be lead back to any element observable through VUV spectroscopy, suggesting that these events are related to low Z materials which are not covered in the spectral range.

Analysis with the HRTS diagnostic on dust particles mobilized by disruptions has revealed that the quantity of dust in the JET-ILW is lower by an order of magnitude than in JET-C. The number of elements detected increases strongly with disruption force, the shape of this distribution with the ILW found to be very similar to that encountered in JET-C. The time evolution of the amount of HRTS dust events is very similar to that of the amount of TIEs the moment the latter are normalized accounting for the observed power threshold. The decrease of a factor $\sim 4$ from the start of the campaign may indicate an erosion or disintegration of the dust particles by contact with the main plasma, the TIEs themselves being the cause of this erosion, or transport to remote and inaccessible areas.

More in-depth analysis of the occurrence of TIEs has to be performed, trying to find a common analysis ground with other diagnostics data as done with the HRTS. Investigating the sizes of the particles may clarify how high the danger of radiative collapse is and comparison of similar statistics from different machines and different divertor geometries could give important information to understand the origins and possible hazardous consequences of such events. 


\section{Acknowledgments}

This work was supported by EURATOM and carried out within the framework of the

European Fusion Development Agreement. The views and opinions expressed herein do not necessarily reflect those of the European Commission.

\section{References}

[1] Winter J 2004 Plasma Physics and Controlled Fusion 46 B583

[2] Roth J et al 2009 Journal of Nuclear Materials 390-391 1 - 9

[3] Endstrasser N et al 2011 Physica Scripta 2011014021

[4] Ekedahl A et al 2009 Journal of Nuclear Materials 390-391 806 - 809

[5] Matthews G 2013 Journal of Nuclear Materials 438, Supplement S2 - S10

[6] Pütterich T et al 2008 Plasma Physics and Controlled Fusion 50085016

[7] Coenen J et al 2013 Nuclear Fusion 53073043

[8] Pasqualotto R et al 2004 Review of Scientific Instruments 75 3891-3893

[9] Giovannozzi E et al 2010 Review of Scientific Instruments 81 10E131

[10] de Vries P C et al 2012 Plasma Physics and Controlled Fusion 54124032

[11] Brezinsek S et al 2013 Journal of Nuclear Materials 438, Supplement S303 - S308

[12] Smirnov R et al 2011 Journal of Nuclear Materials 415 S1067 - S1072 